\title{
Comunicación
}

\section{TROMBOEMBOLISMO AÓRTICO FELINO: RELATO DE CASO}

\author{
Feline Aortic Thromboembolism: Case Report
}

Lizbeth Figueroa A. ${ }^{1}$, Ricardo Paz M. ${ }^{1}$, Diego Díaz C. ${ }^{1}$, Roberto Dávila F. ${ }^{1,2}$

\section{Resumen}

Se presenta el caso de un felino Siamés de 13 años con tromboembolismo aórtico. Esta es una complicación asociada a una cardiomiopatía hipertrófica (CMH), enfermedad cardiaca más común del gato y que se caracteriza por la hipertrofia concéntrica ventricular izquierda.

Palabras clave: tromboembolismo aórtico, $\mathrm{CMH}$, cardiomiopatía felina, coagulopatía

\section{AbSTRACT}

A case of a 13 year old Siamese cat whit feline aortic thromboembolism is presented. This is a devastating complication associated to hypertrophic cardiomyopathy (HCM), one of the most common heart disease in cats characterized by a significant left concentric ventricular hypertrophy.

Keywords: feline aortic thromboembolism, CMH, feline cardiomyopathy, coagulopathies

${ }^{1}$ Clínica de Animales Menores (CAM), Facultad de Medicina Veterinaria, Universidad Nacional Mayor de San Marcos, Lima, Perú

${ }^{2}$ E-mail: rodaf45@yahoo.com

Recibido: 8 de agosto de 2013

Aceptado para publicación: 17 de marzo de 2014 


\section{INTRODUCCIÓN}

El tromboembolismo arterial sistémico (TAS) es una secuela relativamente habitual de la cardiomiopatía hipertrófica felina. A pesar de que puede ocurrir en gatos no cardiópatas y en perros, más del $90 \%$ de los casos que se dan en la práctica clínica afectan a gatos con enfermedad cardíaca (Laste y Haspert, 1995).

La cardiomiopatía hipertrófica es una enfermedad caracterizada por la disfunción diastólica del ventrículo izquierdo como consecuencia de una profusa hipertrofia de la pared, fibrosis miocárdica y, posiblemente, insuficiente perfusión coronaria, determinando que el ventrículo izquierdo se vuelva más rígido y menos distensible. Ante esto, se requiere una mayor presión en el atrio izquierdo para el ventrículo, lo que conduce a una dilatación e hipertrofia del atrio izquierdo. La elevación crónica de la presión diastólica del ventrículo izquierdo se transmite al árbol vascular pulmonar, desencadenando congestión venosa e inclusive edema pulmonar (Kittleson y Kienle, 2000).

Hay tres presentaciones clínicas típicas en gatos con cardiomiopatía hipertrófica: disnea, paresia o parálisis de las extremidades posteriores y muerte súbita. La disnea es atribuible a edema pulmonar y ocasionalmente derrame pleural, en tanto que las dos últimas son el resultado de un tromboembolismo sistémico. Además, estos signos clínicos pueden estar precedidos de otras alteraciones más variables como letargia, tendencia a esconderse, anorexia y vómitos (Mucha y Belerenian, 2008).

La mayoría de los gatos con TAS presentan el trombo alojado en la porción terminal de la aorta abdominal (la trifurcación aórtica) y suele extenderse hacia las arterias ilíacas externas, lo que da el aspecto de una silla de montar (Kittleson y Kienle, 2000). La patogenia de la trombosis comprende una combinación de lesión endotelial, flujo sanguíneo lento y aumento de la coagulabilidad sanguínea, lo que contribuye a la formación de un trombo dentro del atrio izquierdo, y que posteriormente, este coágulo sanguíneo forma un émbolo en la aorta distal (De Francesco, 2003). La explicación más plausible para la formación de trombos en gatos es la lentitud del flujo sanguíneo en la aurícula izquierda (Nelson y Couto, 2010). La presentación clínica más frecuente es un episodio agudo de paresia/parálisis de las extremidades posteriores con dolor extremo, pulso femoral ausente o muy débil, inflamación y contracción de los músculos gastronemios y cianosis de las almohadillas plantares. Son menos frecuentes los signos clínicos referidos a embolización de la arteria braquial derecha, riñones, cerebro, intestino, bazo o hígado (Kittleson y Kienle, 2000).

\section{Presentación del Caso}

\section{Evaluación del Paciente}

Una gata Siamés de 13 años de edad, con $1.96 \mathrm{~kg}$ de peso vivo, procedente del distrito de La Molina, Lima, fue presentado a la Clínica Veterinaria de Animales Menores, Facultad de Medicina Veterinaria, Universidad Nacional Mayor de San Marcos, con historia de tos, vómitos e hiporexia que data desde hace un año.

Al examen clínico, la mascota presentaba signos leves de deshidratación, disnea, tos y taquipnea. Asimismo, taquicardia con sonidos de galope y estertor pulmonar bilateral marcado a la auscultación. El miembro posterior derecho se encontraba rígido e hiperextendido con pérdida de la función motora, frío, sin reflejos, pulso femoral ausente, lechos ungueales pálidos y sin evidencia de sangrado al corte de uña hasta la raíz.

Al examen ecocardiográfico se encontró incrementado el grosor diastólico del septo interventricular $(0.80 \mathrm{~cm})$ y de la pared libre del ventrículo izquierdo $(0.78 \mathrm{~cm})$, reducción de la luz ventricular, así como aumentada la fracción de acortamiento y agrandamiento del atrio izquierdo (Fig. 1). 


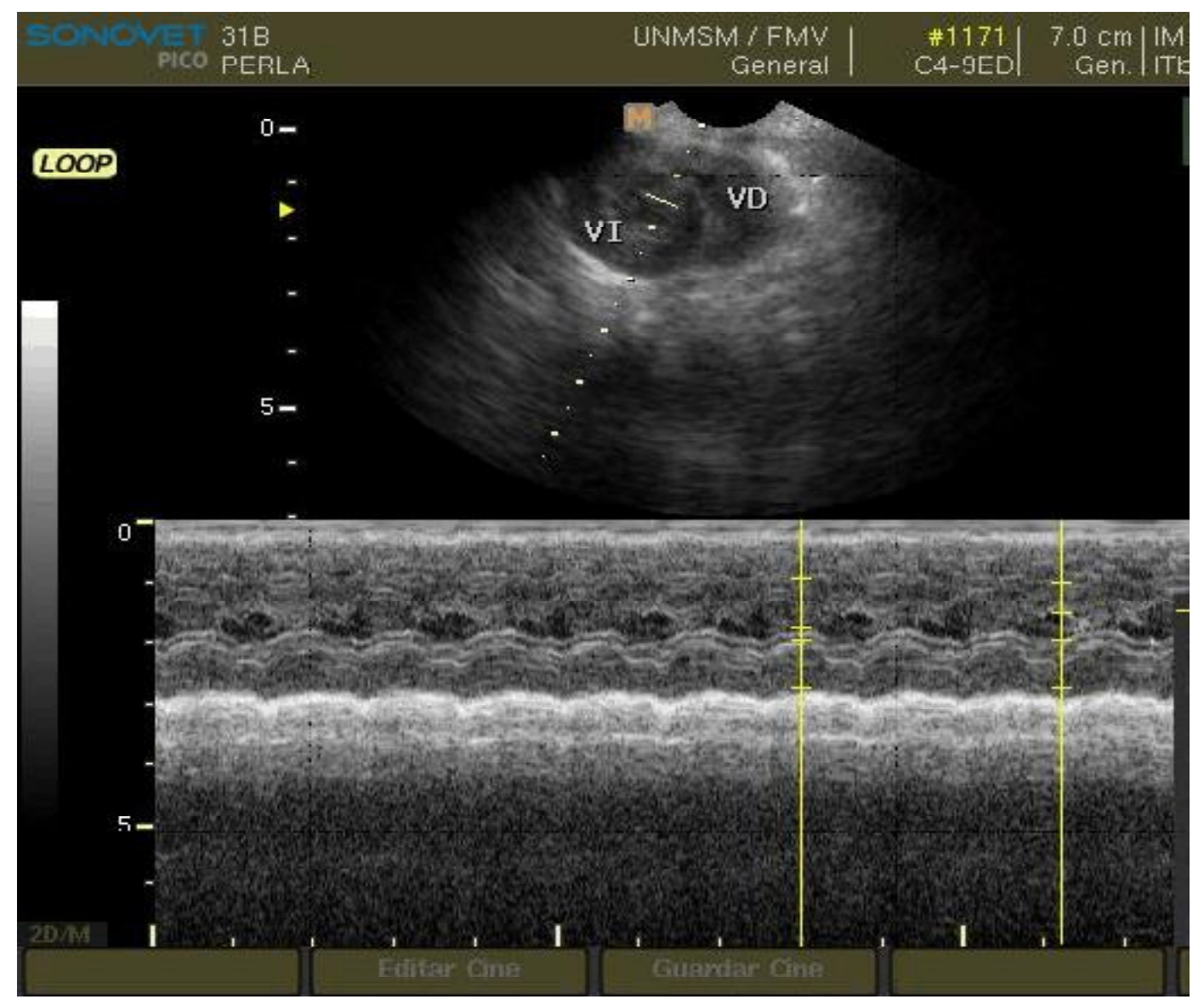

Figura 1. Ecocardiografía de una gata Siamés de 13 años con cardiomiopatía hipertrófica. Se observa un incremento del grosor diastólico del septo interventricular y de la pared libre del ventrículo izquierdo, así como la reducción de la luz ventricular

Al examen radiográfico en la posición lateral derecha del tórax, se observó agrandamiento cardíaco, edema pulmonar y la presencia de una masa en el lóbulo apical que desplazaba la tráquea hacia dorsal. En la vista dorso ventral, el agrandamiento de la aurícula izquierda fue más prominente con un ligero agrandamiento de la silueta cardiaca.

Al examen radiológico contrastado (arteriografía) se evidenció el pasaje del medio de contraste hasta la bifurcación de la aorta, encontrándose obstruido el pase hacia la arteria iliaca del miembro posterior derecho, mientras que pasó con normalidad por todo el paquete vascular del miembro posterior izquierdo (Figura 2).

\section{Tratamiento}

El tratamiento fue orientado a prevenir la formación de futuros trombos, promover la circulación hacia los tejidos isquémicos, manejar el dolor y disolver los émbolos existentes.

Se administró clorhidrato de tramadol en dosis de $4 \mathrm{mg} / \mathrm{kg} \mathrm{SC}$ cada 8 horas, furosemida $4 \mathrm{mg} / \mathrm{kg}$ EV a dosis efecto, heparina $200 \mathrm{U} / \mathrm{kg}$ cada 8 horas y terapia de fluido para reposición con dextrosa al 5\%. Se mantuvo al animal dentro de una cámara de oxígeno, con bolsas de agua caliente y se realizaba masajes periódicos en el miembro afectado. Se indicó 90000 UI EV de estreptoquinasa, pero 


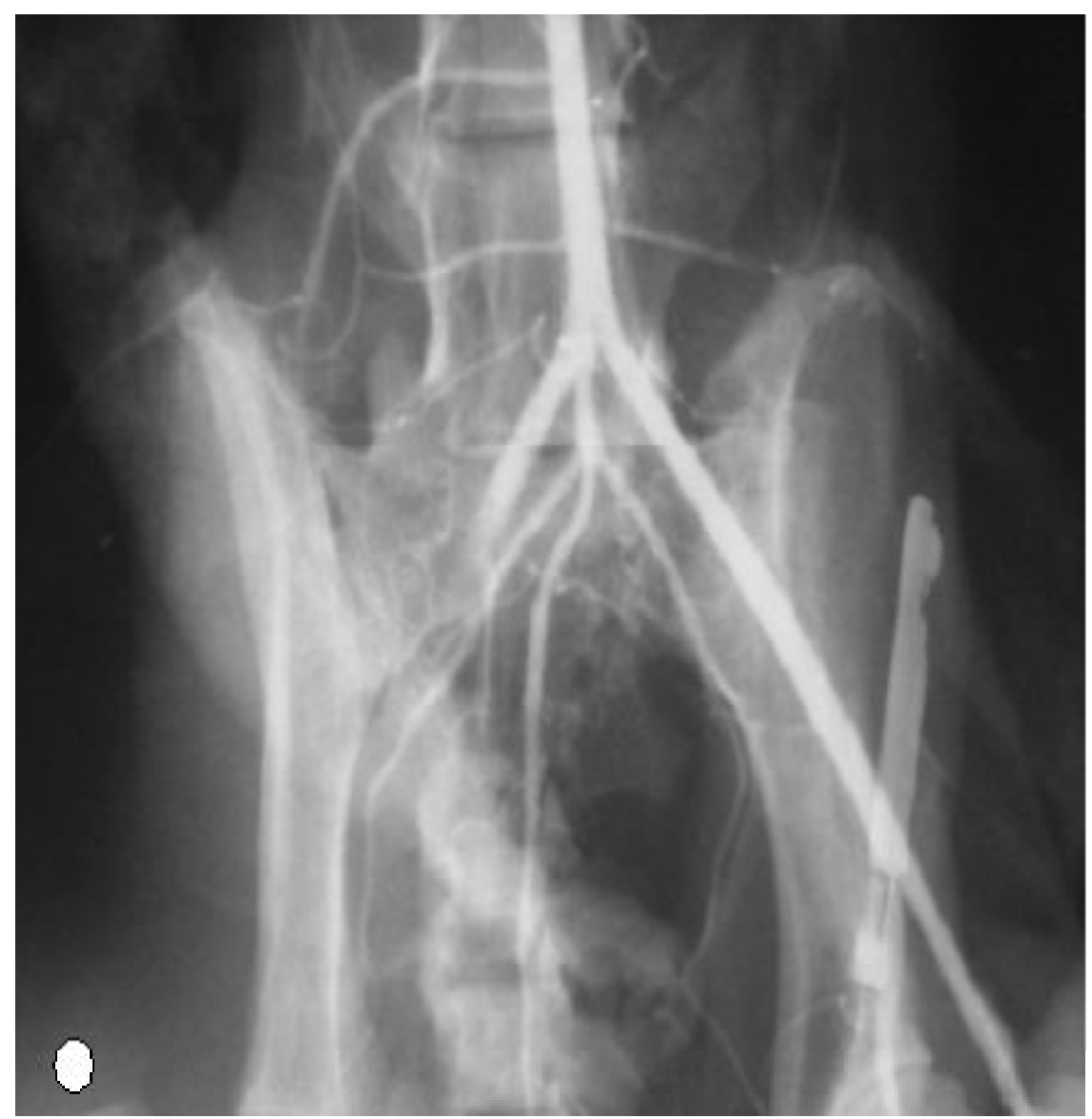

Figura 2. Arteriografía de la cadera de una gata Siamés de 13 años con cardiomiopatía hipertrófica. Obsérvese el bloqueo del pasaje de la sustancia de contraste en la arteria iliaca del miembro posterior derecho. La figura circular marca el lado derecho de la placa radiográfica

el propietario no aceptó al tratamiento por el costo y optó por la eutanasia a las 12 horas de iniciada la atención.

\section{Necropsia}

En la necropsia, la cavidad torácica presentó neoplasias difusas en los lóbulos apical, cardiaco y diafragmático del pulmón derecho y aumento del tamaño cardiaco, principalmente de la aurícula y ventrículo izquierdo. Al corte del corazón se evidenció hipertrofia de la pared ventricular izquierda (Fig. $3)$. Se hizo un corte longitudinal en las arterias aorta e iliacas en la cavidad abdominal y se ubicó el trombo a nivel caudal de la bifurcación de la aorta, en la arteria iliaca derecha (Fig. 4).

\section{Discusión}

El tromboembolismo es una complicación frecuente de la cardiomiopatía hipertrófica felina y de gran riesgo para la vida del paciente. El estasis sanguíneo dentro de las cámaras cardiacas dilatadas y el aumento de la reactividad plaquetaria se combinan y predisponen al gato cardiomiopático al tromboembolismo sistémico (Tilley, 2009). 

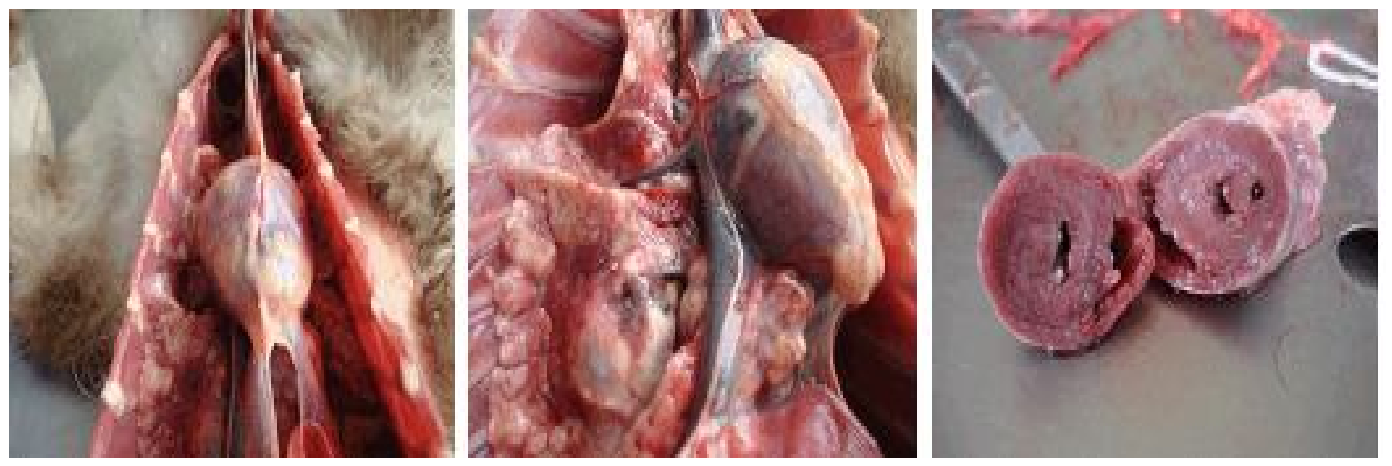

Figura 3. Neoplasias difusas en el pulmón derecho (imágenes del lado izquierdo y del medio). Corte transversal del corazón, donde se aprecia la hipertrofia de la pared ventricular (imagen de la derecha)
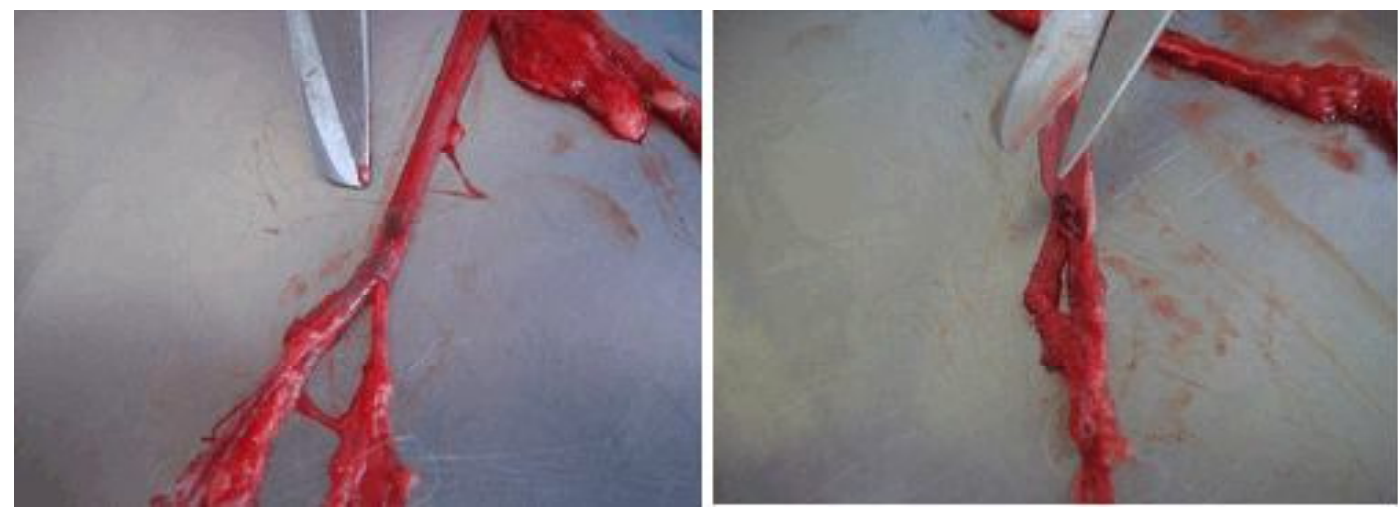

Figura 4. Disección de la arteria aorta y las arterias iliacas, hasta la ubicación del trombo

El examen clínico, radiológico y ecocardiográfico de los gatos con problemas de tos, disnea y vómitos esporádicos es importante para descartar cardiomiopatías y establecer un diagnóstico precoz, a fin de instaurar el tratamiento adecuado (Tilley, 2009). Las alteraciones radiográficas muestran un agrandamiento de las cámaras cardiacas, con edema pulmonar y, a veces, efusión pleural o pericárdica. La ecocardiografía brinda una valoración rápida no invasiva de la estructura y función cardiaca y detecta trombos intracardiacos, lo que posibilita una caracterización precoz y exacta del desorden cardiomiopático (Fox, 1992). El diagnóstico diferencial para la paresia posterior aguda incluye trauma, extrusión discal intervertebral, linfosarcoma raquídeo e infartación fibrocartilaginosa (Nelson y Couto, 2010).

Los objetivos del manejo de gatos con cardiomiopatía hipertrófica incluyen la mejora del llenado ventricular, el control del edema pulmonar o derrame pleural y la prevención de complicaciones tales como el tromboembolismo (Kittleson y Kienle, 2000). Las terapias antiplaquetarias y anticoagulantes se emplean para reducir la agregación plaquetaria y el aumento del tamaño de los trombos existentes. En algunos casos se realiza un tratamiento fibrinolítico (estreptoquinasa, activador del plasminógeno tisular), pero su empleo está limitado debido al alto costo (Nelson y Couto, 2010). 


\section{Literatura Citada}

1. De Francesco TC. 2003. Trombo y embolismo aórtico. En: Tilley LP, Smith FWK (eds). La consulta veterinaria en 5 minutos. Especie canina y felina. $2^{\circ}$ ed. Brasil: Ed Manole. p 450-451.

2. Fox FR 1992. Enfermedades miocárdicas. En: Ettinger SJ (ed). Tratado de medicina interna veterinaria. Tomo II. $3^{\circ}$ ed. Argentina: Ed Intermédica. 2208 p.

3. Kittleson M, Kienle R. 2000. Medicina cardiovascular de pequeños animales. $2^{\circ}$ ed. Barcelona: Ed Gráfica. 672 p.
4. Laste NJ, Haspert NK. 1995: A retrospective study of 100 cases of feline distal aortic thromboembolism: 19771993, J Am Anim Hosp Assoc 31: 492500.

5. Mucha CJ, Belerenian G. 2008. Manual de cardiología veterinaria. Argentina: Holliday-Scott. 113 p.

6. Nelson RW, Couto CG 2010. Medicina interna de pequeños animales. España: Elsevier. 1468 p.

7. Tilley LP. 2009. Tromboembolismo. En: Norsworthy GD et al. (eds). El paciente felino. $3^{\circ}$ ed. Philadelphia, USA: Ed Intermédica. p 329-330. 